\title{
Resti che ritornano testi: il ruolo della memoria in una possibile risemantizzazione di luoghi
}

\author{
di Amedeo Trezza \\ Università di Napoli-amedeotrezza@gmail.com
}

\begin{abstract}
Nowadays, after decades of countryside and agricultural soils abandonment, we are facing more and more some "re-functionalizing" choices. But they too often anticipate a radical loss of sense not followed by a recovery of memory and local identities. In other words, rural architecture and landscape texts are systematically replaced by paralyzing "museifications", accompanied with an idea of "preservation" (with more or less "locked" parks and natural reserves); or by service/industrial aims, or, finally, by tourist based re-interpretations with mass consumerism intentions. Does a 'fourth way' exist which is able to offer a new possibility of meaning to these kinds of territorial texts, without mortifying their memory nor relegating them to a silent role or to "outdated" attitude?

In this perspective of a new kind of possible recuperation, the role of Semiotics could consist in finding out emotional and cognitive valorizations of spatial articulation of sense, as well as vectorial concretions; where policy and architecture could be reciprocally involved towards planning and managing of the territory. In this way the memory of what is left as a mark on the territory of a pre-existent order (already disappeared or disappearing) could offer suggestions to political debates about planning.

In this manner a radical change of the study perspective could be achieved; for instance, manufactures should be assumed not merely as precipitates of ideologies and political strategies but in fact as expressive, material and concrete rests (concretions...) able to activate semiotic processes of recognizing identities and possibilities along with the opening of new meanings.
\end{abstract}

\section{Keywords}

Landscape, Rurality, Memory, Reconversion, Planning

\section{Sommario}

1. Introduzione

2. Fra ideologia e prassi

3. La memoria dei resti e la 'passeggiata' di Pasolini

4. Fiction e reality: a proposito della dialettica di consumo e produzione

5. Memoria del (e nel quotidiano?): un possibile ruolo della semiotica

6. Due possibili risposte: la Valle di Pruno e la Valle d'Aosta

Bibliografia 


\section{Ocula ${ }^{13}$}

Architettura e politica: un incrocio di sguardi | Amedeo Trezza, Resti che ritornano testi: il ruolo della memoria in una possibile risemantizzazione di luoghi.

\section{Introduzione}

Da anni ormai le discipline semiotiche hanno mostrato il loro vivo interesse verso l'architettura, dalle autorevoli voci di Eco e Barthes a partire dagli anni sessanta fino ai più recenti sviluppi della disciplina in questi ultimi anni. Tuttavia è probabile che ciò sia accaduto spesso in maniera discontinua e poco organica anche se ricca ogni volta di nuovi spunti e di validi occasioni di riflessione.

Far triangolare semiotica e architettura con la politica - o meglio, far dialogare politica e architettura osservando questo dialogo da una prospettiva semiotica - può essere di certo una tappa importante di questo percorso interdisciplinare di ricerca, nella misura in cui la pratica architettonica può essere certo l'estrinsecarsi di scelte politiche come di esigenze sociologiche ma al contempo valere anche come preziosa cartina di tornasole quanto agli effetti che tali scelte o esigenze hanno saputo o dovuto ingenerare, nel bene come nel male, nei territori abitati dall'uomo, condivisi o contesi.

Se però il dibattito contemporaneo sull'architettura non è certo nuovo alle attenzioni sugli esiti delle dinamiche di incontro/scontro tra architettura e politica, il compito - e il presunto e tutto da dimostrare valore aggiunto della semiotica in merito a tale dibattito potrebbe essere a mio avviso duplice. Anzitutto metodologico: assumere le due discipline come discorsi semiotici specifici e osservare quindi il rapporto tra essi come un discorso di/su discorsi, ovvero un meta-discorso sul senso dell'abitare e di gestire lo spazio condiviso e la cosa pubblica. In seconda istanza la semiotica, arricchita dall'esperienza di queste pratiche di gestione del senso potrebbe offrire loro - a queste pratiche - gli strumenti per interrogarsi sul loro reciproco ingaggio, ovvero tentando di far luce sui meccanismi di influenza reciproca, mettendo sotto osservazione le rispettive pratiche di utilizzo dello spazio e dei manufatti.

Coerentemente con queste riflessioni, nelle prossime pagine cercherò di avanzare l'ipotesi secondo cui i testi paesaggistici e architettonici non sarebbero più da assumersi solo come precipitati di ideologie e di strategie politiche, poiché dopo esser stati tali appaiono in seguito - ad uno sguardo altro, successivo - piuttosto come resti significanti, materici, che rilanciano la scommessa del senso guardando ora di nuovo alla politica, costringendo cioè il suo sguardo (quello della politica) a soffermarsi su di essi (i resti) e a prendere atto della loro semplice presenza (residualità) e allo stesso tempo suggerendo all'opinione pubblica - così come ai gestori ed ai pianificatori di turno - un nuovo punto di partenza, talvolta finanche provocatorio, per nuove strategie di inerenza dell'uomo al mondo, ovvero per immaginare nuove ripartenze, a partire proprio da quelle forme residuali, testimonianze indiziarie. 


\section{Ocula ${ }^{13}$}

Architettura e politica: un incrocio di sguardi | Amedeo Trezza, Resti che ritornano testi: il ruolo della memoria in una possibile risemantizzazione di luoghi.

\section{Fra ideologia e prassi}

Ma facciamo un passo indietro. Immaginando un'autorevole critica voce semiotica in merito al rapporto tra ideologia, potere e architettura, credo sia difficile prescindere dal tono delle sia pur note considerazioni lotmaniane a proposito della città di Pietroburgo. Entrando nel merito dell'idea di una "dialogicità" produttiva, il semiologo russo ravvisa nel carattere simbolico della funzione architettonica e nella spazialità intesa come "parametro" d'interazione i criteri utili affinché ogni dialogo interdisciplinare sulle città non resti sterile:

Sicuramente lo spazio rappresenta il più profondo, ma non l'unico, parametro del contesto cittadino. Anche gli edifici reali, se acquistano un carattere simbolico, diventano suoi elementi. [...] Il contesto non è monolitico, anch'esso al suo interno è attraversato da dialoghi. Gli edifici costruiti in epoche differenti, spesso molto remote, formano nel loro funzionamento culturale un'unità. La diversità di epoca crea la varietà, mentre la stabilità degli archetipi semiotici e della serie delle funzioni culturali garantiscono l'unità. Il questo caso l'insieme si forma in modo organico, non come esito del progetto di un qualche costruttore, ma come realizzazione di spontanee tendenze della cultura (Lotman 1998: 47).

È chiaro come Lotman utilizzi per la sua classificazione tipologica dello spazio abitato le categorie di una semiotica della cultura che gli consentono d'intravedere le regole ispiratrici dell'urbanistica nel susseguirsi delle epoche come emanazioni più o meno occasionali derivanti dai complessi sistemi socio-culturali che le hanno generate, all'interno di orizzonti concettuali ben precisi e identificabili, anche se in continua evoluzione.

Diventa molto interessante a questo punto ricordare come lo stesso autore legge due importanti testi urbanistici - Mosca e Pietroburgo - in cui emergenze ideologiche di carattere politico si esprimono attraverso impianti urbanistici specifici se pur in modo affatto differenti tra essi:

Il punto di vista (il vettore di orientamento spaziale) di Pietroburgo è lo sguardo di un pedone che cammina in mezzo alla strada (il soldato in marcia). È innanzitutto la prospettiva aperta, diritta: nel XVIII secolo i viali (prospekty) si chiamavano proprio così, "prospettive" (perspektivy) [...]. Lo spazio era orientato. Era delimitato ai lati dalle masse nere delle case e illuminato da entrambi i lati dalla luce delle notti bianche. [...] Ma la prospettiva si estendeva proprio da Oriente a Occidente e, nel periodo delle notti bianche, a una delle estremità splendeva sempre l'alba, conferendo alla strada un'apertura cosmica (prima la via si chiamava "delle albe rosse"). [...] Se Mosca tende tutta a un centro comune (il Cremlino, centro dei centri che sovrasta i centri locali delle chiese), Pietroburgo è tutta proiettata fuori di sé, è una strada, una "finestra sull'Europa". L'introduzione di linee verticali, che richiedono uno sguardo dal basso verso l'alto, si contrappone al contesto pietroburghese. [...] L'orientamento spaziale della vecchia Mosca coincideva con lo sguardo di un pedone che percorresse le curve dei vicoli. Chiese e villette ruotavano davanti ai suoi occhi come su una piattaforma teatrale. Non una precisa silhouette, ma un gioco di superfici. Lo spazio di Pietroburgo, come una scenografia teatrale, non 


\section{Ocula ${ }^{13}$}

Architettura e politica: un incrocio di sguardi | Amedeo Trezza, Resti che ritornano testi: il ruolo della memoria in una possibile risemantizzazione di luoghi.

ha il fondale, quello moscovita non ha la facciata principale. L'ampliamento e il raddrizzamento delle vie moscovite ha eliminato questo gioco spaziale. Il contesto (lo spirito della città) è prima di tutto la sua struttura generale (Lotman 1998: 46-47).

Questa apertura lotmaniana, sebbene pure datata, riesce ancora a introdurre la possibilità di una prospettiva di indagine semiotica rivolta proprio a un tema nel tema, così come anche esplicitamente suggerito dall'occasione di questi scritti semiotici sul rapporto tra architettura e politica: ovvero problematizzare il tema della memoria nelle articolazioni tridimensionali del senso nello spazio, nell'accezione che a questo termine verrà attribuita qui di seguito.

\section{La memoria dei resti e la 'passeggiata' di Pasolini}

A seguito delle necessarie considerazioni introduttive, ci pare altrettanto necessaria una precisa delimitazione del campo di indagine. Parlare del rapporto tra architettura e politica in generale (sebbene sub specie semiotica) può risultare tanto improduttivo quanto fuorviante. Restringiamo pertanto il nostro campo di applicazione alle architetture rurali ed ai paesaggi agricoli (e alle loro possibili destinazioni d'uso) rispetto alle varie scelte rifunzionalizzanti che paiono oggi obbligate (perlopiù di carattere industriale, turistico e terziaristico, come vedremo) dagli esiti socioeconomici e culturali di un'era contemporanea essenzialmente postindustriale, cifra di tempi 'moderni' che solo formalmente paiono essere meno autoritari delle prospettive totalitaristiche a cui prima con Lotman si è fatto esplicito richiamo.

Come sappiamo, col boom industriale degli anni cinquanta e sessanta a cui è seguita - pare come logica conseguenza - l'avvento della società dei consumi che abbiamo visto consolidarsi negli ultimi decenni del secolo scorso, le politiche dei paesi industrializzati hanno consegnato all'uomo del nuovo millennio territori nazionali (in Italia, in particolar modo) invasi dalla cementificazione a scopo residenziale, industriale e terziaristico in cui appaiono del tutto residuali gli spazi agricoli e rurali, se non riconvertiti in agricoltura meccanizzata (come appare evidente nelle grosse pianure del nord Italia, e in misura minore nel Tavoliere, nella Terra di lavoro e nell'Agro Pontino).

Inevitabilmente a questi territori residuali (nell'ottica dello sfruttamento massivo delle risorse) nel migliore dei casi è stato affidato, col tempo, il compito di custodi della memoria di un tempo passato e superato. Una memoria, come vedremo subito, spesso contesa e resa poi anche strumentale a quelle stesse logiche che l'hanno associata a brandelli di territorio sparsi qua e là sulla penisola, per circoscrivere il fenomeno al nostro paese.

Quando Pasolini, nel documentario Rai trasmesso il 7 febbraio del 1974 (Pasolini 1974), passeggiava tra le dune di Sabaudia denunciando l'appiattimento culturale operato dalla società dei consumi più ancora che dai regimi totalitari, aveva ben presente il potere dirompente di un sistema socio-economico che, più sottilmente ma molto più efficacemente di intenti 


\section{Ocula ${ }^{13}$}

Architettura e politica: un incrocio di sguardi | Amedeo Trezza, Resti che ritornano testi: il ruolo della memoria in una possibile risemantizzazione di luoghi.

politici dichiarati (come nelle città sovietiche di lotmaniana memoria), era riuscito a destrutturare l'economia profonda - quella emotiva e delle relazioni, dei desideri oltre che quella dei bisogni - degli italiani.

La realtà che la società dei consumi dei "regimi democratici" ha minato alle fondamenta è stata, dice Pasolini, la «realtà dell'Italia provinciale, rustica, paleo-industriale» perché, continua, «quella acculturazione, quella omologazione che il fascismo non è riuscito assolutamente a ottenere, il potere della civiltà dei consumi, invece, riesce a ottenere perfettamente, distruggendo le varie realtà particolari, togliendo realtà ai vari modi di essere uomini che l'Italia ha prodotto in modo storicamente molto differenziato» (Pasolini 1974).

Ciò che di sostanzialmente irreversibile è accaduto nella gestione del territorio rurale italiano negli ultimi decenni, non è tanto una sovrapposizione di stili o di costruiti incoerenti con le preesistenze dettata da esigenze produttive ma una molto più profonda rivoluzione dei comportamenti delle popolazioni, da cui sono scaturiti come logica conseguenza altrettanti comportamenti le cui tracce si leggono sui territori rurali che ora, col senno di poi, ci appaiono devastati.

Ma è proprio il "senno di poi" che può, se non davvero assennato, completare l'opera distruttiva attraverso una mistificazione di ciò che resta, una funzionalizzazione di quei resti, scampati forse per miracolo, del tutto fuorviante e ancora una volta funzionale a quella stessa logica del consumo di cui sopra.

\section{Fiction e reality: a proposito della dialettica di consumo e produzione}

Infatti sempre più assistiamo oggi, a fronte di un pluridecennale abbandono delle campagne e dei terreni agricoli, a ben poche scelte rifunzionalizzanti che troppo spesso preludono a desemantizzazioni radicali senza che ad esse faccia seguito un recupero della memoria e delle identità locali. In altri termini, testi architettonici e paesaggistici rurali lasciano sistematicamente il posto o a museificazioni cristallizzanti a scopo tutelativo (parchi e riserve più o meno "blindati"), oppure a destinazioni terziaristiche $\mathrm{o}$ industriali $\mathrm{o}$, in fine, a reinterpretazioni in chiave turistica di matrice consumistica di massa, che pure è una pratica semiotica interessante (Brucculeri 2009). Esiste invece una "quarta via" che possa offrire a tali porzioni testuali di realtà una possibilità di senso che non mortifichi la memoria senza però essere al contempo "fuori moda" o essere relegata solo, nel migliore dei casi, a un "fuori onda"?

Fatte salve le riconversioni di terreni e contesti agricoli e rurali in siti industriali o residenziali, per i quali l'abbandono della destinazione funzionale d'origine è dichiarata, evidente e in alcuni casi irreversibile, a ben vedere le atre due vie - quella tutelativa, che abbiamo chiamato "museale", e quella turistica, invece "speculativa" - soggiacciono alla medesima logica rifunzionalizzante, seppur con strategie diverse. 


\section{Ocula ${ }^{13}$}

Architettura e politica: un incrocio di sguardi | Amedeo Trezza, Resti che ritornano testi: il ruolo della memoria in una possibile risemantizzazione di luoghi.

Giustapposte una tutela rigorosa, ad esempio, di una ZPS (zona a protezione speciale) di un parco nazionale e un moderno villaggio turistico nato sulle rovine di una antica masseria, queste due scelte rifunzionalizzanti di un territorio paiono essere agli antipodi. E lo sono. Tuttavia ritengo che siano gli esiti più lontani tra loro di un medesimo atteggiamento nei confronti della ruralità: entrambi scaturiti dalla presa d'atto di un distacco irreversibile dal flusso della quotidianità che per secoli e millenni ha segnato e interpretato i luoghi scelti; entrambi gli atteggiamenti immaginano gli ex territori rurali come simulacri di un mondo perduto.

E ciò può accadere e di fatto accade proprio in virtù di quella destrutturazione e di quel riposizionamento del sistema dei comportamenti della nostra società rispetto a quella tradizionale, processi - come detto prima, di "economia profonda" - in virtù dei quali noi ora sentiamo quei testi paesaggistici talvolta con benemerenza, talaltra con sufficienza, più spesso con distacco, ma comunque e in ogni caso lontani.

Laddove la destinazione turistica (con varie declinazioni consumistiche e approcci culturali, dall'agriturismo al residence di lusso, dal campeggio alla country house) propone al consumatore una fiction di un mondo perduto o ideale che non esiste (ad esempio la spiaggia incontaminata, le acque pulitte e pescose, attraverso tutto un modo di gestione del soggiorno improntato ad una rivendicata genuinità e tipicità dei prodotti offerti, a partire dalla promozione pubblicitaria, per continuare al soggiorno vero e proprio e per finire alle foto ricordo), la destinazione museale propone al consumatore visitatore, turista - diciamo così - soft, un discorso diverso che più che una fiction propone piuttosto un reality di un mondo che non c'è più ma che si presume riesumare o difendere nelle sue ultime fattezze. Certo, il ruolo dei parchi e delle ZPS è importante e utile per indiscutibili ragioni ecologiche e biologiche che non staremo qui a discutere né tantomeno ad elencare, ma a patto che queste soluzioni non si riducano a musei a cielo aperto, a prigioni senza sbarre, che non siano una nuova e raffinata forma per negare ancora una volta all'uomo il contesto vitale e produttivo da cui proviene e che lo ha generato.

Produttivo, appunto. Perché proprio in questo i due estremi si toccano: entrambe le soluzioni, quella turistica - a vario grado - e quella tutelativa anche qui a vario grado - credo siano sfumature a loro volta graduali di un unico atteggiamento: quello di considerare l'uomo/fruitore solo e soltanto come consumatore di un prodotto da offrire e non più anche e soprattutto come produttore di un bene comune e condivisibile, ruolo che invece offre e sintetizza l'orizzonte socio-culturale ed economico delle società rurali (Petrini 2005).

Fino a che l'uomo resta consumatore e non produce più perché il meccanismo della delega della produzione è affidata ad una non meglio precisata alterità (ora ad esempio le province dello sfruttamento cinese e domani chissà), non credo si possa uscire da questo circolo vizioso che non sa proporci realtà ma solo, a seconda dei casi, della sua fiction o del suo reality, laddove per fiction sarà da intendersi l'esibizione di una finzione (consapevolmente riconosciuta tale dal lettore/fruitore) di una realtà altra non più reperibile (per scelta o per necessità) e laddove invece per reality si 


\section{Ocula ${ }^{13}$}

Architettura e politica: un incrocio di sguardi | Amedeo Trezza, Resti che ritornano testi: il ruolo della memoria in una possibile risemantizzazione di luoghi.

intenderà quel meccanismo - spesso perverso e ambiguo nel rapporto tra autore e fruitore - attraverso il quale si vorrà proporre un testo complesso polisensoriale e multistratificato (nel nostro caso un testo paesaggistico o architettonico) come se fosse vero - nel senso di originale -, spiato senza che questo sguardo furtivo di chi spia infici la sua naturalezza, dando adito a credere che le cose stiano proprio così.

E allora, da un lato: chi produrrà più cosa e dove quando ogni territorio sarà sede possibile per un periodo di vacanza, una vacatio planetaria? In un mondo vacante? E dall'altro: che destino può avere a lungo termine la logica della tutela integrale dei parchi naturali se nel frattempo le conurbazioni delle megalopoli di tutto il mondo si espandono a dismisura e la popolazione mondiale cresce vertiginosamente? È possibile e utile pensare a delle concrete risposte?

Non sarebbe più utile che le aree non ancora compromesse anziché blindarle inutilmente siano invece l'occasione per offrire (molto più utilmente) al resto del territorio un possibile modello di sviluppo sostenibile non solo economicamente ed ecologicamente, ma anche e soprattutto culturalmente, lavorando quindi proprio su un nuovo riposizionamento e ripensamento del sistema dei comportamenti umani?

\section{Memoria del (e nel quotidiano?): un possibile ruolo della semiotica}

Di fronte alla prospettiva di tali questioni il ruolo della semiotica potrebbe consistere nell'individuare le valorizzazioni emotive e cognitive dell'articolazione dello spazio (degli spazi) come vere e proprie concrezioni vettoriali di senso (dove si gioca la partita delle istanze politiche verso la pianificazione e la gestione del territorio) e anche viceversa (l'architettura verso la politica) nella misura in cui il portato memoriale di ciò che resta come traccia sul territorio di un ordine pregresso (già scomparso o in via di disparizione) possa suggerire invece stimoli ai dibattiti politici in tema di pianificazione e di riqualificazione.

Quanto ai paesaggi rurali gli oggetti del contendere tra pianificazione e reinterpretazione dei luoghi paiono essere dunque i terreni agricoli dismessi, le infrastrutture rurali in abbandono, i ruderi di vecchi fabbricati rurali. Questi resti dicono di un passato diverso e scomparso ma di questo passato sono altamente informativi: il terreno agricolo dismesso ormai improduttivo $\mathrm{e}$ in via di rinaturalizzazione conserva delle tracce della sua funzionalità $\mathrm{e}$ delle sue coltivazioni (vecchi alberi, tracce di piante rinate spontaneamente, inselvatichite); le infrastrutture in abbandono mostrano residui di canalizzazioni e raccolta delle acque, sistemi di tenuta e fertilizzazione del terreno con terrazzamenti, dissodamenti del terreno, pozzi; infine i ruderi, perlopiù stalle o vecchie case agricole che a mala pena mostrano ancora in piedi le pareti perimetrali con qualche logora architrave in legno. In questo momento storico dell'evoluzione del nostro paese abbiamo ancora la possibilità di osservare la presenza significativa nelle campagne delle forme 


\section{Ocula ${ }^{13}$}

Architettura e politica: un incrocio di sguardi | Amedeo Trezza, Resti che ritornano testi: il ruolo della memoria in una possibile risemantizzazione di luoghi.

di queste architetture rurali che, sebbene in rapido disfacimento, in alcuni casi mostrano ancora bene le proprie fattezze.

Ebbene, questi ruderi sono dei resti di un sistema di vita e di gestione del territorio non più praticato, sono resti abbandonati e disaggregati che scompaiono lentamente ma inesorabilmente. Tuttavia sono dei testimoni (e in quanto tali dei testi complessi) di un ordine socio-culturale che ci siamo lasciati alle spalle. Questi resti, nonostante la loro caducità, resistono ancora ai nostri occhi, a noi che non li utilizziamo più, e dunque, a dispetto del tempo, restano. E ci parlano.

Proprio in quanto defunzionalizzate tali strutture di senso ora riescono a parlarci ancor meglio poiché sono diventate dei segni di loro stesse, o ancor più dei segni metonimici di un intero e complesso mondo di cui sono gli ultimi testimoni. Il sacrificio dell'utilizzo - l'inutilizzo attuale - ha permesso loro di parlarci, li ha caricati di una facoltà simbolica tale da averli trasformati in oggetti sociali e politici del contendere (dalla tutela integrale ai più disparati tentativi di riqualificazione).

Sono diventati dei nodi di senso altamente significativi per la nostra società attraverso cui passano decisioni e intendimenti, diciamo così, interdisciplinari. La politica, più o meno strumentalmente, si interessa a loro nella gestione del territorio chiamando in causa pianificatori e urbanisti che, anch'essi più o meno benevolmente, sono chiamati a decretarne le sorti (nella migliore delle ipotesi, se non a consegnarli definitivamente all'oblio e alla disparizione).

È in questo senso che, come si è detto prima, il ruolo della semiotica - in quanto semiotica del testo paesaggistico - risiede a mio avviso in due momenti: anzitutto in una preventiva individuazione di concrezioni vettoriali di senso, un senso dei luoghi convenzionalmente e comunitariamente stabilito e ad essi attribuito. Attraverso questa individuazione è possibile poi riconoscere quei nodi di senso attraverso i quali si articolano le negoziazioni del senso, generate da dinamiche di interazioni tra attribuzioni di valore ai manufatti e a porzioni di spazio. Le diverse valorizzazioni emotive e cognitive assegnate a porzioni di mondo dai corpi sociali che abitano un territorio, a loro volta frutto del successo o dell'insuccesso di pratiche di utilizzo di quelle porzioni, innescano contese di riconoscimenti e disconoscimenti dei luoghi abitati, o abbandonati.

Stando così le cose, per ritornare alle pagine introduttive di questo scritto e volendo ora individuare un secondo momento di un possibile ruolo della semiotica nella dinamica di incontro-scontro tra discipline, si attuerebbe un ribaltamento della prospettiva d'indagine nella misura in cui i manufatti non sarebbero più da assumersi solo come precipitati di ideologie e di strategie politiche (valenza connotativa) ma proprio invece come resti significanti, materici, concreti (appunto concrezioni...) che proprio in quanto tali rimettono in moto il processo semiotico di riconoscimento delle identità e delle possibilità aprendo nuove scommesse di senso.

Siamo perlopiù abituati a pensare, infatti, nei termini lotmaniani del caso riportato in apertura, nella misura in cui assetti ideologici e interpretativi generano assetti territoriali in qualche modo a loro immagine e somiglianza (e fortemente connotati). A questa visione ingenua (e univoca) dei rapporti 


\section{Ocula $\mathbf{a}^{13}$}

Architettura e politica: un incrocio di sguardi | Amedeo Trezza, Resti che ritornano testi: il ruolo della memoria in una possibile risemantizzazione di luoghi.

sociali e della gestione comunitaria dei luoghi facilmente si sostituisce invece una interpretazione interattiva maggiormente complessa: non solo i luoghi abitati ed i manufatti architettonici a loro volta generano e orientano "di ritorno" le pratiche di utilizzo dei corpi sociali orientandone scelte e convinzioni, ma possono fare anche di più.

In altri termini non assistiamo di certo ad un monologo della politica nei confronti delle pratiche architettoniche ma i testi-manufatti realizzati, come ogni testo, una volta realizzati vivono di una vita loro propria dagli esiti inediti per chiunque, tanto più per gli autori e per i loro mandanti (politici) che non hanno certo più il potere e l'autorità di condizionarne l'utilizzo che può assumere - e spesso lo fa - risvolti affatto inediti.

Il monologo si fa dialogo. Anzi di più, multiforme e imprevedibile andirivieni in un mosaico di "pratiche quotidiane" (de Certeau 2001) che mostrano una incalcolabilità di comportamenti e di destinazioni (di "usi") a cui un manufatto culturale è sottoposto:

In realtà, a una produzione razionalizzata, espansionista, centralizzata, spettacolare e chiassosa, fa fronte una produzione di tipo diverso, definita "consumo", contrassegnata dalle sue astuzie, dalla sua frammentazione legata alle occasioni, dai suoi bracconaggi, dalla sua clandestinità, dal suo instancabile mormorio, che la rende quasi invisibile poiché non si segnala in alcun modo attraverso creazioni proprie, bensì mediante un'arte di utilizzare ciò che le viene imposto. [...] Così il successo spettacolare della colonizzazione spagnola fra gli indios è stato svuotato dall'uso che se n'è fatto: benché sottomessi, se non addirittura consenzienti, spesso questi indigeni usavano le leggi, le pratiche o le rappresentazioni loro imposte con la forza o con la seduzione per finalità diverse da quelle dei conquistatori; le trasformavano in qualcosa d'altro,se sovvertivano dall'interno [...]. Essi metaforizzavano così l'ordine dominante: lo facevano funzionare secondo un altro registro. [...] Lo aggiravano senza sottrarvisi [...] (de Certeau 2009: 66-67).

Nel caso dei resti, dei ruderi - per tornare al nostro tema specifico assistiamo, come si è detto, a un meccanismo del tutto simile. Pratiche d'uso sotterranee rinnovano il potenziale semantico di questi testi architettonici, li reinterpretano secondo direzioni e vettori di senso alquanto inediti e ogni volta diversi, anche imprevedibili.

Inoltre questi precipitati abitativi e costruttivi invecchiati e decadenti non solo hanno svolto a loro tempo il ruolo di testi architettonici fortemente connotati in un certo modo, come si è visto poc'anzi, quando cioè erano segni vivi di un utilizzo declinato al tempo presente in seno ad una società rurale in ottima salute, ma ora, in quanto segni ultimi di un assetto territoriale, politico e sociale scomparso, diventano super-segni (direbbe il geografo e paesaggista Turri, degli iconemi) di quel mondo scomparso (Turri 2004). Detto in termini semiotici, si caricano ancor più di un valore memoriale e sono ancor più connotati.

$\mathrm{Ma}$, e qui sta a mio avviso la cifra di questa indagine, tali super-segni sanno diventare allo stesso tempo testimoni di loro stessi, affatto autoreferenziali, e sanno emergere in tutta la loro diretta e immediata funzione direi più che testimoniale, quasi testamentaria, rendendosi 


\section{Ocula ${ }^{13}$}

Architettura e politica: un incrocio di sguardi | Amedeo Trezza, Resti che ritornano testi: il ruolo della memoria in una possibile risemantizzazione di luoghi.

trasparenti alla materia con la quale sono stati costruiti, facendo cioè trasparire la materia (talvolta destrutturata se non anche rinaturalizzata) nella sua immediatezza, puro denotatum.

Questa presentificazione della materia, di una materia trattata dall'uomo e ora ritrattata lentamente dal tempo, risulta di certo fortemente informativa agli occhi dell'architetto e del pianificatore dal punto di vista della memoria storica e collettiva di un luogo e di un corpo sociale, carica di rimandi e di significazioni che eccedono la sua inconsistente durabilità, ma - di nuovo allo stesso tempo proprio in quanto residuo materiale, in quanto traccia materica, puro volume, nella sua semplice datità immediata di corpo fisico, si propone come resto. $\mathrm{E}$ in quanto resto si pone come un problema irrisolto e da risolvere. Non è niente, resta comunque qualcosa, un dato di fatto nei confronti del quale bisogna prendere delle decisioni. Un "residuo fisso" dal quale ripartire, rispetto al quale ri-prendere posizione, appunto riposizionarsi.

È propriamente qui che si attua quel ribaltamento di prospettive di interazione tra le discipline, è qui che l'architettura rilancia alla politica la scommessa del senso. Questi suoi segni materici devono interrogare la gestione della cosa pubblica e fare problema alla politica e/o alla opinione pubblica, costringerle a compiere delle scelte. E tutto non può avvenire che attraverso la semiotica. O meglio, tale ribaltamento interattivo di discipline pare avere un suo agio argomentativo e una sua libertà di movimento interpretativo proprio attraverso un approccio di carattere semiotico.

\section{Due possibili risposte: la Valle di Pruno e la Valle d'Aosta}

Al fine di suggerire eventuali modelli di risposte alle questioni avanzate fino ad ora, mi pare possa essere utile accennare ad alcuni possibili esempi concreti attraverso i quali possiamo forse intravedere dei tentativi di reazione nei confronti di paesaggi agricoli e di architetture rurali (nel primo caso la politica accanto alla opinione pubblica e nell'altro invece in assenza della politica) che vadano già al di là del turismo consumistico o museale, della industrializzazione o della tutela stretta. I due casi a cui voglio fare riferimento sono per tanti motivi lontani geograficamente e culturalmente tra loro e infatti offrono spunti differenti e hanno obiettivi differenti. Tuttavia mi pare rappresentino due fenomeni molto importanti e interessanti.

6a. Il primo interessa la valle di Pruno, sita nei pressi del Monte Cervati, all'interno della Zona A del Parco Nazionale del Cilento e del Vallo di Diano, in provincia di Salerno. Le antiche comunità prunesi erano dislocate in tre comuni vicini alla valle (Piaggine, Laurino e Rofrano) ed erano dedite, come del resto tutte le popolazioni non solo del Cilento interno ma pressoché di tutto l'Appennino italiano, prevalentemente all'agricoltura e alla pastorizia fin da epoche remote. La valle di Pruno inoltre era nota al Regno delle Due Sicilie come florido granaio poiché la particolare esposizione e il suo clima hanno consentito per secoli l'affermazione della coltura del grano. Ancora 


\section{Ocula ${ }^{13}$}

Architettura e politica: un incrocio di sguardi | Amedeo Trezza, Resti che ritornano testi: il ruolo della memoria in una possibile risemantizzazione di luoghi.

oggi il territorio vanta alcuni grani antichi non compromessi geneticamente, così come altra zone rurali del Cilento e del Meridione, anche se la produzione di grano non è più la caratteristica di questa zona montuosa del Cilento che ha subito a più riprese e con varie vicissitudini la sorte di quasi tutti i territori agricoli del Mezzogiorno: l'emigrazione e l'abbandono (Roberto 2009).

Negli anni del secolo scorso l'abbandono ha creato oblio in molti casi e silenzio, perdita di saperi e di memoria collettiva, di cultura. In questi ultimi anni però, ad opera di un gruppo di persone (l'Associazione L.O.S.A.P.) è stato avviato un progetto spontaneo e partecipato di recupero di questi antichi grani altrimenti destinati alla scomparsa e attraverso studi e stanziamenti fisici in loco, piccoli investimenti, in valle si è ricominciato a coltivare i grani antichi e a riappropriarsi produttivamente dei terreni abbandonati. Ancora pochi. Anche se la scommessa è tutt'altro che vinta a causa infatti di forti resistenze culturali della popolazione originaria residente in parte aspra e ostile e della scarsa collaborazione fattiva delle istituzioni locali, negli ultimi anni sono aumentati gli ettari di terra piantati a grano, si è creata una forma interessante di ricettività partecipativa selezionata a chi è interessato alla realtà che va ad incontrare ed è iniziato un percorso di recupero culturale e memoriale (Avagliano 2009). Recentemente è stato anche installato un nuovo mulino di fattura moderna ma di concezione tradizionale destinato alla molitura dei grani prodotti sul posto al fine di ottenere un macinato di alta qualità attraverso un sistema alternativo alla molitura industriale, un prodotto finito con un valore aggiunto sia in termini organolettici e nutritivi che culturali e sociali.

Tuttavia l'isolamento socio-culturale continua ad essere forte e ad oggi una esperienza simile non può dirsi ancora compiuta e quindi non può ancora essere un modello da esportare, sebbene non per suo demerito ma per evidenti motivazioni di carattere contestuale.

Ciononostante, quel che differenzia questa esperienza da tante altre solo apparentemente simili consiste in un fattore importantissimo: non si tratta di una attività economica fine a se stessa e con l'obiettivo di fare business essendole sotteso un progetto culturale e di vita. Il recupero delle colture tradizionali cerealicole è alla base di una concezione del mondo e dell'economia alternativa a quella del sistema dominante: si produce per la sussistenza, si auto-produce. Il prodotto finito, sebbene abbia un valore quantificabile economicamente, non ha come fine quello di essere piazzato sul mercato globale tra tanti altri ma quello di essere un tassello nell'economia alimentare di una comunità umana legata alla terra e che dalla terra vuole trarre sostentamento e nutrimento senza sfruttarla con concezioni industriali. Per una volta il prodotto è visto dal punto di vista del produttore e non da quello del consumatore, o meglio il consumatore coincide col (e presuppone il) produttore, anche simbolicamente.

Facilmente intuibili gli impatti architettonici e paesaggistici sul territorio, gli effetti di tale progetto complesso e coraggioso sono tutt'altro che invasivi o mortificanti: il recupero delle architetture tradizionali per essere effettivamente riutilizzate, il recupero di valli ed ettari di terreno non più abbandonati alla vegetazione spontanea e di nuovo coltivati puntando sulla 


\section{Ocula ${ }^{13}$}

Architettura e politica: un incrocio di sguardi | Amedeo Trezza, Resti che ritornano testi: il ruolo della memoria in una possibile risemantizzazione di luoghi.

qualità e non meccanicamente sulla quantità, la riappropriazione pedonale di nodi strategici quali antiche vie, sentieri, passaggi forestali, sorgenti, greti di torrenti e così via.

Inoltre, ciò che distingue tale progetto dai recuperi a scopo turistico, oltre all'effettivo utilizzo a scopo produttivo di tali siti e manufatti, è un nuovo modo di concepire l'agricoltura come lavoro e prospettiva per il futuro e che però allo stesso tempo non sia né una fiction, né un reality, attrattive simulacrali per turisti in erba, né tantomeno agricoltura industriale, bensì produzione di beni di prima necessità usando, utilizzando il territorio, sebbene esso sia ricadente in una zona A di un parco nazionale.

Tali complesse attività reticolari sul territorio lo ridisegnano proprio a partire da quei resti di cui prima si diceva e si propongono come risposte concrete ad un dialogo troppe volte infecondo tra politica e architettura, questa volta spontaneo e fatto da pratiche semiotiche (Fontanille 2008) che contribuiscono a ridisegnare e a ri-organizzare (nel senso di rendere di nuovo organica) attraverso una doppia inscrizione reciproca (dell'uomo sul territorio e del territorio sull'uomo) un nuovo e si spera durevole esempio di semiosfera (Lotman 1985).

6b. Il secondo esempio che intendiamo offrire all'attenzione dei lettori è di tutt'altro spirito e fattezze, sebbene le finalità a lungo termine possano in parte coincidere. Si tratta di un complesso e articolato progetto di recupero dei paesaggi rurali tradizionali, degli usi, dei costumi e della lingua delle popolazioni autoctone di quel settore alpino che interessa la Valle D'Aosta al confine col Piemonte e con le Alpi francesi ad ovest (Savoie e Haute Savoie). Col patrocinio, l'interesse e le partecipazioni della Soprintendenza regionale per i Beni Culturali della Regione Valle d'Aosta, dell'Assessorato all'Educazione e alla Cultura, del Boureau Régional pour l'Ethnologie et la Linguistique, dell'Assessorato dell'Agricolura e nell'ambito del progetto comunitario transfrontaliero italo-francese (Interreg III A 2000-2006 ITALIE-FRANCE) denominato Paysages...à croquer, l'Associazione Musei di Cogne, la Fondation Grand Paradis e la partecipazione spontanea di molti abitanti di questi luoghi - ciascuno a vario titolo - hanno realizzato in questi ultimi anni delle importanti ricerche socio-culturali, linguistiche, architettoniche a antropologiche sul territorio di appartenenza.

Di particolare interesse per il nostro tema è un preciso settore di ricerca praticato, quello teso al recupero della memoria locale attraverso l'architettura rurale della Valle d'Aosta. Come si legge in una recente pubblicazione: "Dal 1960 in poi, la comunità valdostana ha conosciuto profondi mutamenti: la crisi dell'agricoltura e della società agropastorale, i cambiamenti demografici, lo sviluppo dell'industria e successivamente del settore terziario, soprattutto del turismo, l'onnipresenza dei mass-media, l'urbanizzazione estrema, con la conseguente rapida espansione che ha trasformato i comuni di periferia in dormitorio delle città [...]. Dagli anni Sessanta, invece, in Valle si constata una vera e propria interruzione della continuità culturale, una frattura netta tra il prima e il dopo. Tutto è cambiato: le gerarchie sociali, le regole di comportamento, le abitudini linguistiche, la composizione etnica [...]" (B.R.E.L. 2005: 11). 


\section{Ocula ${ }^{13}$}

Architettura e politica: un incrocio di sguardi | Amedeo Trezza, Resti che ritornano testi: il ruolo della memoria in una possibile risemantizzazione di luoghi.

Alla luce di queste considerazioni, a partire dal 1987 la Soprintendenza regionale per i Beni Culturali della Valle d'Aosta ha proceduto al censimento dell'architettura rurale tradizionale di tutta la regione. Questa importante attività ha permesso di acquisire in maniera scientifica e sistematica la memoria dei luoghi e delle tecniche costruttive tradizionali, pubblicando anche un interessante lavoro in proposito (Association des Musées de Cogne: 1997). Tra i vari risultati conseguiti questo interesse fattivo ha permesso di ristrutturare due antiche case rurali assorte a testimonianze-prototipo dell'architettura rurale tradizionale valdostana: la Maison de Cogne a Cogne che ospita manifestazioni, eventi, rassegne ed esposizioni collegate alle attività di ricerca e di promozione della Valle e la Maison Bruil a Introd, antica casa rurale a funzioni concentrate risalente al 1683 ed oggi rifunzionalizzata ad eco-museo a scopo didattico all'interno del quale sono state riprodotte in maniera esplicativa le funzioni e le destinazioni originarie di ciascun ambiente della complessa struttura rurale: dalla crotta alla ghiacciaia naturale, alle zone di essiccazione.

Parallelamente a questa iniziativa della Soprintendenza, inoltre, l'Associazione dei Musei di Cogne ed il Bureau Régional pour l'Ethnologie et la Linguistique ha svolto un encomiabile lavoro di raccolta sul campo di testimonianze orali e di documenti scritti riguardanti la vita rurale tradizionale degli abitanti della Valle, con interessantissimi risvolti linguistici (tutela e studio del Patois, dialetto transfrontaliero locale) e dalla preziosa catalogazione di testimonianze sulla conservazione e trasformazione dei prodotti agricoli di una volta. Anche da questa iniziativa è nata una pubblicazione che raccoglie a scopo divulgativo il materiale testimoniale (B.R.E.L. 2005).

6c. Anche in questo caso, sebbene in modi diversi, si supera la concezione di sfruttamento turistico senza ricorrere necessariamente alla tutela integrale, anzi promuovendo circolazione di competenze e di saperi tesi ad una riqualificazione del mondo rurale in chiave contemporanea e non nostalgico-archivistica, utilizzando fattivamente un territorio senza imbalsamarlo né snaturarlo e al contempo generando a latere anche un interesse turistico di qualità, senza che questo diventi però il fine ultimo.

\section{Conclusione}

Esperienze molto diverse tra loro (anche se solo si osserva il coinvolgimento e l'interesse della popolazione e delle istituzioni nei due casi) quella cilentana e valdostana sono esempi di quanto sia importante reinterpretare il patrimonio culturale e produttivo del mondo agricolo per il futuro della nostra società veloce e fragile. Quanto, inoltre, sia fecondo e qualificante escogitare una "quarta via" - come si diceva all'inizio - per riscoprire un sano dialogo tra architettura e politica.

E pare che proprio alle prese con esempi di tal fatta, con esperimenti quali li abbiamo visti in queste pagine, possano emergere i nodi problematici 


\section{Ocula $\mathbf{a}^{13}$}

Architettura e politica: un incrocio di sguardi | Amedeo Trezza, Resti che ritornano testi: il ruolo della memoria in una possibile risemantizzazione di luoghi.

e al contempo le potenzialità di uno scambio di saperi e di saper-fare tra queste due discipline, entrambe ad alto potenziale simbolico.

\section{Bibliografia}

Association des Musées de Cogne,

1997 Architecture Rurale en Vallée d'Aoste. La maison de Cogne (Quaderni 2), Sarre (AO), Imprimerie Testolin.

Avagliano, A.,

2009 Attacca u' padrone addo' vole-a u' ciuccio, Sapri, L.O.S.A.P.

B.R.E.L.,

2005 Conserver le souvenir...se souvenir pour conserver, Aosta, Arti Grafiche E. Duc Aoste.

Brucculeri, M. C.

2009 Semiotica per il turismo, Roma, Carocci.

Certeau de, M.

2009 L'invenzione del quotidiano, Roma, Edizioni Lavoro.

Fontanille, J.

2008 Pratiques sémiotiques, Paris, Puf.

Lotman, J. M.

1985 La semiosfera, Venezia, Marsilio.

1998 Il girotondo delle muse, Bergamo, Moretti \& Vitali.

Pasolini, P.

1974 "Pasolini e ... la forma della città", dalla serie Io e..., documentari a cura di Brunatto P., Roma, Teche RAI.

Petrini, C.

2005 Buono, pulito e giusto, Torino, Einaudi.

Roberto, F.

2009 Pruno (1880-2007). Una comunità che scompare. Senza rimpianti? Sapri, Tipografia MDD.

Turri, E.

2004 Il paesaggio e il silenzio, Venezia, Marsilio. 